\title{
Effect of IRAK1 on Apoptosis and Necroptosis of Hepatoma Cell Line SK-Hep1
}

\author{
Zhijia Pan, Xiaolei Zhang, Changshi Qian, Xiaochen Liu, Xinglin Jin* \\ Department of Hepatopancreatobiliary Surgery, Affiliated Hospital of Yanbian University, Yanji, China \\ Email: *xljinyj@163.com
}

How to cite this paper: Pan, Z.J.., Zhang, X.L., Qian, C.S., Liu, X.C. and Jin, X.L. (2019) Effect of IRAK1 on Apoptosis and Necroptosis of Hepatoma Cell Line SK-Hep1. Chinese Medicine, 10, 19-29.

https://doi.org/10.4236/cm.2019.101003

Received: December 19, 2018

Accepted: February 10, 2019

Published: February 13, 2019

Copyright $\odot 2019$ by author(s) and Scientific Research Publishing Inc. This work is licensed under the Creative Commons Attribution International License (CC BY 4.0).

http://creativecommons.org/licenses/by/4.0/

\begin{abstract}
Interleukin I receptor associated kinase 1 (IRAK1) is a downstream signal molecule of activated MyD88 recruitment, which can activate Fas associated death domain protein (FADD) to induce apoptosis. IRAK1 can also activate tumor necrosis factor-related factor 6 (TRAF6) and induce the expression of a series of downstream specific genes. IRAK1 is an essential factor in the induction of mitochondrial division and necroptosis. In the current study, RNAi technique was used to silence IRAK1, and the apoptosis and necroptosis rate of SK-Hep1 cells were detected by flow cytometry. The apoptosis and the necroptosis pathway of hepatoma SK-Hep1 cells were blocked separately, and the expressions of FADD, RIP1 and TRAF6 genes were silenced separately. The results showed when the expression of IRAK1 was down-regulated, the apoptosis and necroptosis rate of SK-Hep1 cells were significantly increased. With silenced FADD, RIP1 and TRAF6, respectively, the expression of IRAK1 protein had no significant change. However, the expression of IRAK1 mRNA decreased significantly $(\mathrm{p}<0.01)$ after the silencing of RIP1 and TRAF6 genes, while the IRAK1 mRNA did not change significantly after the silencing of FADD genes; when $\mathrm{z}$-VAD-FMK was interfered, the expression of IRAK1 mRNA decreased significantly after the silencing of TRAF6 genes, while the IRAK1 mRNA did not change significantly after the silencing of FADD and RIP1genes. The study shows that RAK1 gene inhibits apoptosis and necroptosis in SK-Hep1 cells. TRAF6 gene affected the role of IRAK1 in apoptosis and necroptosis, RIP1 gene affected the role of IRAK1 in apoptosis, while FADD gene did not affect the role of IRAK1 in apoptosis and necroptosis.
\end{abstract}

\section{Keywords}

IRAK1, Apoptosis, Necroptosis, FADD, RIP1, TRAF6 


\section{Introduction}

Most primary hepatocellular carcinoma (HCC) is associated with inflammation and cirrhosis [1] [2] [3] [4] [5]. Chronic injury and inflammation are closely related to tumorigenesis [6]. TLR4 in TLRs family is one of the important members that regulate the immune response and inflammatory reaction. TLR4 activates downstream signal transduction elements through two downstream pathways MyD88-dependent and MyD88-independent to induce apoptosis and necroptosis [7]. IRAK1 is a downstream signal molecule of activated MyD88 recruitment, which can activate FADD to induce apoptosis. IRAK1 can also activate TRAF6 to induce the expression of downstream specific genes. IRAK1 is an essential factor in the induction of mitochondrial division and necroptosis [8]. Receptor-interacting protein-1 (RIP1) is a downstream signaling molecule in the MyD88-independent pathway, which is not only a necessary condition for initiating necroptosis, but also a key factor for inhibiting necroptosis [9]. RIP1 limits caspase-dependent tumor necrosis factor (TNF)-induced apoptosis and is also involved in receptor-interacting protein-3 (RIP3)-dependent TNF-induced necroptosis [10]. Decreasing the expression of IRAK1 can inhibit the activity of ERK signaling pathway, reduce the activity of NF-kB, and inhibit the proliferation of mesenchymal stem cells [11].

In this study, we have carried out related experiments on SK-Hep1 hepatoma cells in vitro to clarify the effect of IRAK1 on apoptosis and necroptosis, and the relationship between IRAK1, FADD, RIP1 and TRAF6 in the process of apoptosis and necroptosis.

\section{Materials and Methods}

Cell lines and main reagents. The human hepatoma cell line SK-Hep1 was purchased from the cell bank of the Chinese Academy of Sciences. Necrostatin-1 (Nec-1) was purchased from Sigma, USA, z-VAD-FMK inhibitor was purchased from Biyuntian Biotechnology Company, China. FADD siRNA, RIP1 siRNA, TRAF6 siRNA were purchased from Shanghai Jima Pharmaceutical Technology Company, China. IRAK1 siRNA was purchased from Guangzhou Ruibo Biotechnology Company, China. The antibody was purchased from Elabscience Biotechnology Company, China. The primer was purchased from Invitrogen Biotechnology Company, China.

Cell culture and treatment. SK-Hep1 cells were cultured in DMEM medium containing $10 \%$ fetal bovine serum (FBS) at $37^{\circ} \mathrm{C}$ and $5 \% \mathrm{CO}_{2}$. When the cells were adherent to the wall and grew to more than $80 \%$, the cells were subcultured. The $3 \times 10^{5}$ cells were inoculated into the six-well plate and cultured for $24 \mathrm{~h}$ after adding Nec- 1 and $\mathrm{z}$-VAD-FMK.

MTS method. The DMEM containing 10\% fetal bovine serum (FBS) and SK-Hep1 cells were fully mixed and inoculated into 96-well plates $\left(10^{4}\right.$ cells/well). The cells were cultured in $5 \% \mathrm{CO}_{2}$ incubator at $37^{\circ} \mathrm{C}$ for 24 hours, then Nec-1 $(30 \mu \mathrm{M}, 60 \mu \mathrm{M}, 90 \mu \mathrm{M})$ and z-VAD-FMK $(10 \mu \mathrm{M}, 20 \mu \mathrm{M}, 40 \mu \mathrm{M})$,) 
were added to culture for 24 hours. The cells were treated with MTS cell proliferation kit (solarbio, China) for $3 \mathrm{~h}$ (20 L/well). The wavelength of $490 \mathrm{~nm}$ was selected to determine the light absorption (OD) value of each well. The cell survival rate was calculated, and the optimal concentration of $\mathrm{Nec}-1$ and $\mathrm{z}$-VAD-FMK was screened.

Flow cytometry. Annexin V-FITC/PI kit was used for (BD, USA) detection. The cells treated with IRAK1siRNA for $48 \mathrm{~h}$ were washed twice with precooled PBS, then resuspended with $100 \mu \mathrm{L}$ Binding Buffer and collected, about $3 \times 10^{5}$ cells per tube. After $5 \mu \mathrm{L}$ Annexin V-FITC was added, the mixture was well mixed, and the mixture was kept at room temperature for $15 \mathrm{~min}$, followed by the addition of $5 \mu \mathrm{L}$ PI and $400 \mu \mathrm{L}$ Binding Buffer. Apoptosis rate and necrotic apoptosis rate were detected by FACSCalibur (BD, USA).

Gene transfection. Each siRNA $10 \mu \mathrm{l}$ and $10 \mu \mathrm{l}$ Lipofectamine TM 2000 (Invitrogen, USA) were added into the blank DMEM medium to mix well. After 20 min, the cells were incubated in $37^{\circ} \mathrm{C}$ incubator for $6 \mathrm{~h}$, then replaced with $10 \%$ FBS in DMEM medium for $48 \mathrm{~h}$. The transfection effect was observed. The siRNAs sequence is as follows:

FADD: 5'-GCCUAGACCUCUUCUCCAUTT-3';

RIP1: 5'-CCUUCUGAGCAGCUUGAUUTT-3';

TRAF6: 5'-GUCGCCUUGUAAGACAAGATT-3';

IRAK1: 5'-GAGCCACCGCAGATTATCA-3';

Negative Control: 5'-UUCUCCGAACGUGUCACGUTT-3'.

Western blot method. The total protein was extracted by RIPA Lysis Buffer (Solarbio, China) and quantified by (Solarbio, China) with BCA protein analysis kit. Different concentrations of SDS-PAGE gels were prepared according to the molecular weight of various proteins and separated by $100 \mathrm{~V}$ constant pressure electrophoresis apparatus. Then transferred to PDVF membrane for $1.5 \mathrm{~h}$ and $10 \%$ skimmed milk powder for $1 \mathrm{~h}$, then added the first antibody (FADD, RIP1, TRAF6, IRAK1 at 1:1000, $\beta$-actin at 1:2000), incubated at $4^{\circ} \mathrm{C}$ overnight, washed with TBST for 3 times, then added the second antibody (prepared at 1:1000), incubated at room temperature for $1 \mathrm{~h}$, washed 3 times by TBST. The ECL luminescent chromogenic liquid (Solarbio, China), was prepared according to 1:1 ratio. The PDVF film was soaked for $5 \mathrm{~min}$. The film was photographed and analyzed by FluorChem HD2 and FC2 software (ProteinSimple, USA).

RT-PCR method. Total RNA was collected by Trizol reagent (Life Technologies, USA) and extracted. After sample quantification, cDNA, was synthesized by reverse transcription using synthetic kit (Promega, USA) and amplified by Green Supermix (Promega, USA) in a specific PCR cycle. The expression of mRNA was detected by gel electrophoresis. The sequence of primers is as follows:

GAPDH forward 5'-ACCACAGTCCATGCCATCAC-3', reverse 5'-TCCACCACCCTGTTGCTGTA-3';

FADD forward 5'-GCTCAAGTTCCTATGCCTCG-3', 
reverse 5'-TGACGTTAAATGCTGCACAC-3';

RIP1 forward 5'-GGCTTTGGGAAGGTGTCTCT-3',

reverse 5'-TCATCTTCGCCTCCTCCAAG-3';

TRAF6 forward 5'-AGCTCCTGTAGCGCTGTAAC-3', reverse 5'-CTGGACATTTGTGACCTGCAT-3';

IRAK1 forward 5'-GAGAAGCACCCAGAGCACAC-3', reverse 5'-CTGCCTGCAGCTTCTCTAGC-3'.

Statistical analysis. SPSS 17.0 software and GraphPad Prism 7.0 software were used to analyze the data. The measurement results were expressed as mean \pm standard deviation, and the difference was statistically significant by $\mathrm{t}$-test $(\mathrm{P}<$ 0.05).

\section{Results}

Optimum concentration of Nec-1 and $z$-VAD-FMK. Hepatoma SK-Hep1 cells were treated with different concentrations of Nec-1 $(30 \mu \mathrm{M}, 60 \mu \mathrm{M}, 90 \mu \mathrm{M})$ and Z-vad-fmk (10 $\mu \mathrm{M}, 20 \mu \mathrm{M}, 40 \mu \mathrm{M})$ ), for $24 \mathrm{~h}$. OD values were detected and the relative survival rates were calculated. When the concentration of Nec-1 was 60 $\mu \mathrm{M}$, the OD value and the cell survival rate were the highest. When the concentration of Z-vad-fmk was $20 \mu \mathrm{M}$, the OD value and the cell survival rate were the highest. Therefore, $60 \mu \mathrm{M}$ of Nec-1 and $20 \mu \mathrm{M}$ of Z-VAD-FMK were selected to interfere with the cells, see Table 1.

Effect of IRAK1 on apoptosis and necrotic apoptosis of SK-Hep1 cells. IRAK1siRNA was transfected into SK-Hep1 cells, and the effect of transfection was detected by RT-PCR method. The expression of IRAK1 mRNA was significantly decreased $(P<0.05)$. See Figure 1 . Furthermore, apoptosis rate and necroptosis rate were detected by flow cytometry. The results showed that the apoptosis rate was $1.53 \%$ in blank transfection group, $14.93 \%$ in IRAK1siRNA transfection group, the necroptosis rate was $3.08 \%$ in blank transfection group and $18.23 \%$ in IRAK1siRNA transfection group. The differences of apoptosis rate and necroptosis rate were significantly increased compared with the blank group $(\mathrm{P}<0.01)$. The results showed that IRAK1 could promote the apoptosis and necroptosis in SK-Hep1 cells, see Figure 2.

Relationship between expression of IRAK1 protein and three genes of FADD, RIP1 and TRAF6. The expression of three proteins was detected by Western Blot after FADDsiRNA, RIP1siRNA and TRAF6siRNA were transfected into SK-Hep1 cells. The results showed that all three proteins were significantly decreased $(\mathrm{p}<$

Table 1. OD (490 nm) and CSR of SK-Hep1 cells treated with Nec-1 and z-VAD-FMK for $24 \mathrm{~h}$.

\begin{tabular}{cccccccc}
\hline & & DMSO & & Nec-1 $(\mu \mathrm{M})$ & & \multicolumn{2}{c}{ Z-VAD-FMK $(\mu \mathrm{M})$} \\
\cline { 3 - 7 } & negative & $(2 \mu \mathrm{L} / \mathrm{ml})$ & 30 & 60 & 90 & 10 & 20 \\
\hline OD & $0.81 \pm 0.02$ & $0.80 \pm 0.01$ & $0.85 \pm 0.02$ & $0.87 \pm 0.03^{*}$ & $0.84 \pm 0.03$ & $0.73 \pm 0.03$ & $0.75 \pm 0.06^{*}$ \\
CSR & $100.00 \%$ & $98.11 \%$ & $104.72 \%$ & $107.27 \%$ & $103.53 \%$ & $91.10 \%$ & $92.58 \%$ \\
\hline
\end{tabular}

CSR: cell survival rate, ${ }^{*}$ compared with DMSO group, $\mathrm{P}<0.05$. 


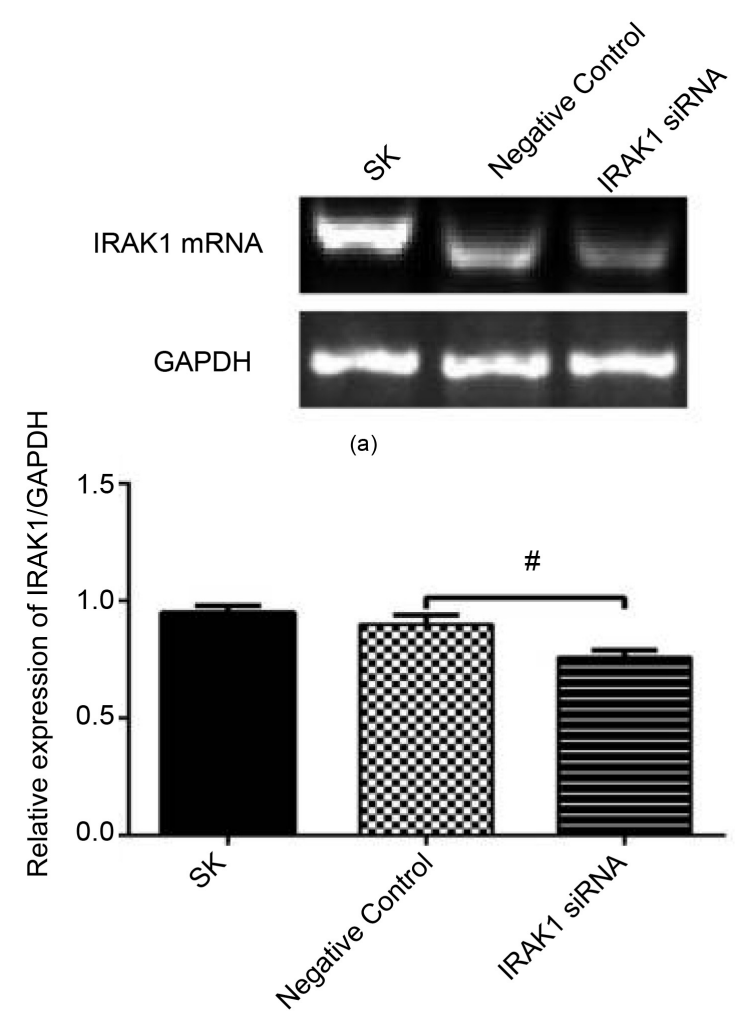

(b)

Figure 1. (a) RT-PCR assay was used to detect the expression of IRAK1 mRNA in SK-Hepl cells transfected with IRAK1siRNA; (b) Compared with blank siRNA group, the expression of IRAK1 mRNA decreased significantly after successful transfection $(\#$ : $\mathrm{P}<0.05)$.

0.01), see Figure 3, indicating that the transfection was successful. Furthermore, $60 \mu \mathrm{M}$ of Nec-1 and $20 \mu \mathrm{M}$ of $\mathrm{z}$-VAD-FMK were used to detect the expression of IRAK1 protein in SK-Hep1 cells with silenced FADD, RIP1 and TRAF6, respectively. The results showed that the expression of IRAK1 protein in SK-Hep1 cells had no significant change ( $\mathrm{p}>0.05$ ), see Figure 4 and Figure 5.

The relationship between the expression of IRAK1 $\mathrm{mRNA}$ and three genes of FADD, RIP1 and TRAF6. With $60 \mu \mathrm{M}$ of Nec-1 and $20 \mu \mathrm{M}$ of z-VAD-FMK were used to detect the expression of IRAK1 mRNA in SK-Hep1 cells in silenced FADD, RIP1 and TRAF6, respectively. After Nec-1 intervention, the expression of IRAK1 mRNA in hepatoma SK-Hep1 cells transfected with RIP1siRNA and TRAF6siRNA decreased significantly $(\mathrm{p}<0.01)$, while the expression of IRAK1 mRNA in hepatoma SK-Hep1 cells transfected with FADDsiRNA did not change significantly ( $p>0.05$ ), when $z$-VAD-FMK was added, the expression of IRAK1 mRNA decreased significantly $(\mathrm{p}<0.01)$. The expression of IRAK1 mRNA in hepatoma SK-Hep1 cells transfected with TRAF6siRNA decreased significantly $(\mathrm{p}<0.01$ ), while the expression of IRAK1 mRNA in hepatoma SK-Hep1 cells transfected with FADDsiRNA and RIP1siRNA did not change 


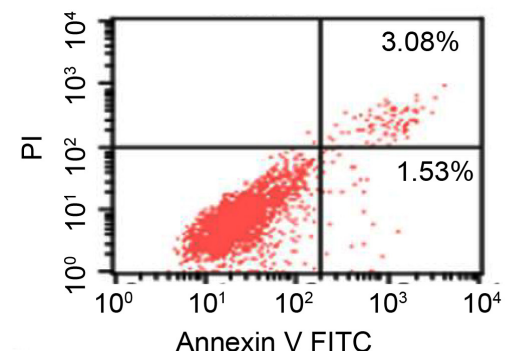

(a)

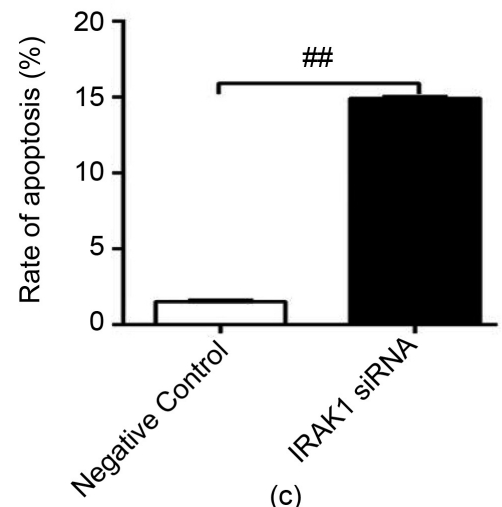

(c)

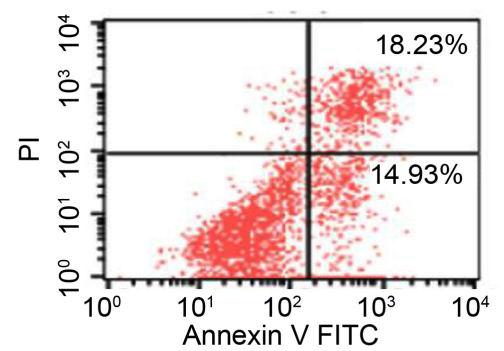

(b)

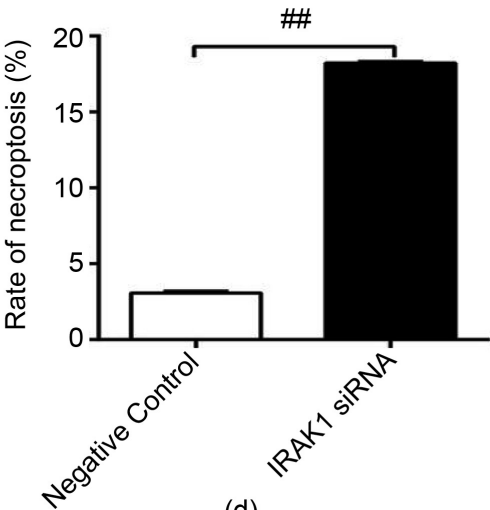

(d)

Figure 2. Apoptosis and necroptosis rate of SK-Hep1 cells transfected with IRAK1siRNA were detected by flow cytometry. (a) The apoptotic rate of transfected blank siRNA group was $1.53 \%$, necroptotic rate was $3.08 \%$; (b) The apoptotic rate of transfected IRAK1siRNA group was $14.93 \%$, necroptotic rate was $18.23 \%$; (c) The apoptotic rate of transfected IRAK1siRNAgroup was significantly higher than that of transfected blank siRNA group; (d) the necroptotic rate of transfected IRAK1siRNA group was significantly higher than that of transfected blank siRNA group. (\#: $\mathrm{P}<0.01$ ).

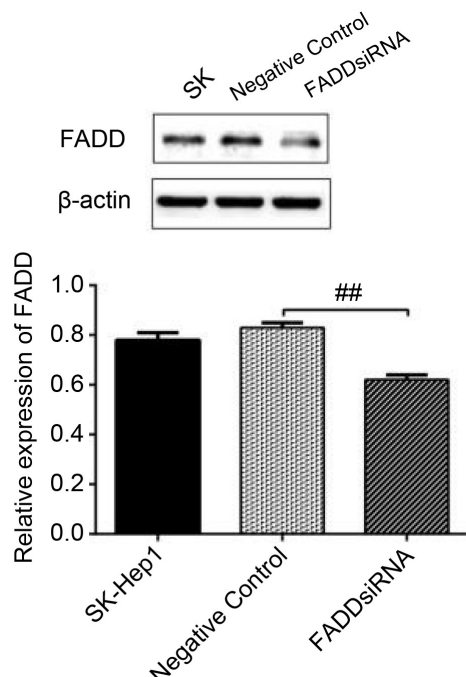

(a)

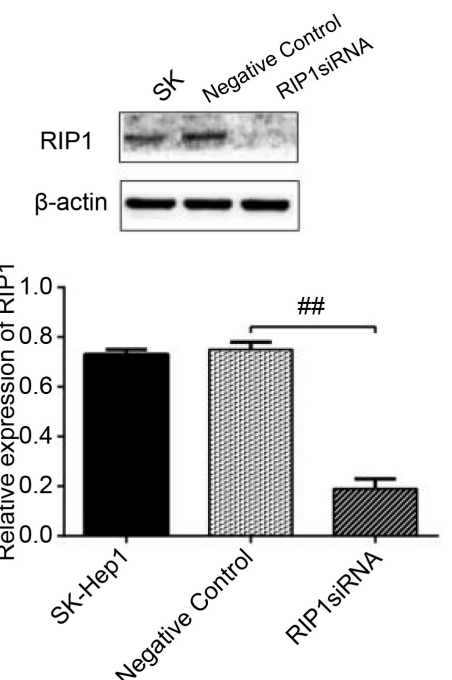

(b)

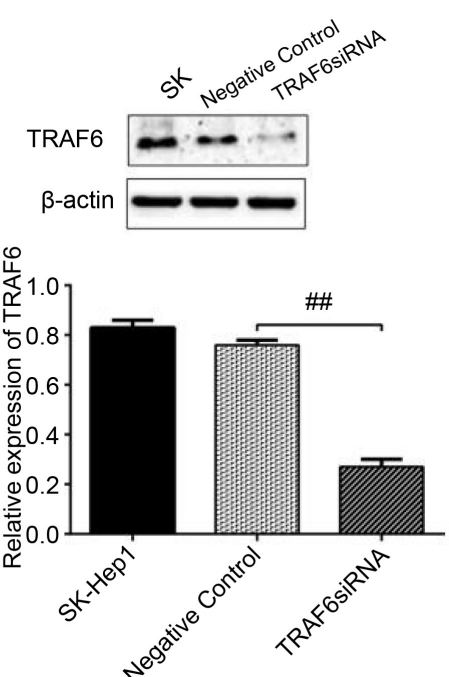

(c)

Figure 3. Western blot was used to detect gene silencing after transfection with FADD, RIP1 and TRAF6. (a) After transfection of FADDsiRNA, the expression of FADD protein was significantly weaker than that of the blank group; (b) After transfection of RIP1siRNA, the expression of RIP1 protein was significantly weaker than that of the blank group; (c) After transfection of TRAF6siRNA, the expression of TRAF6 protein was significantly weaker than that of the blank group. (\#\#: $\mathrm{P}<0.01)$. 


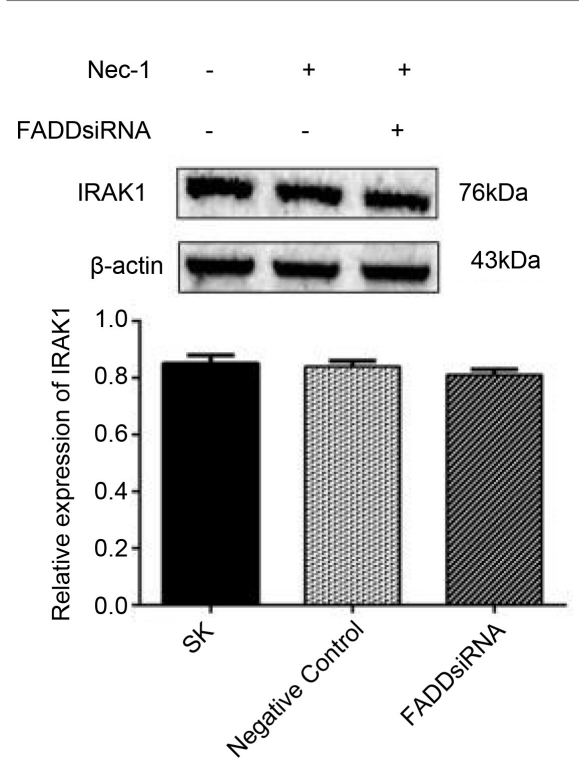

(a)

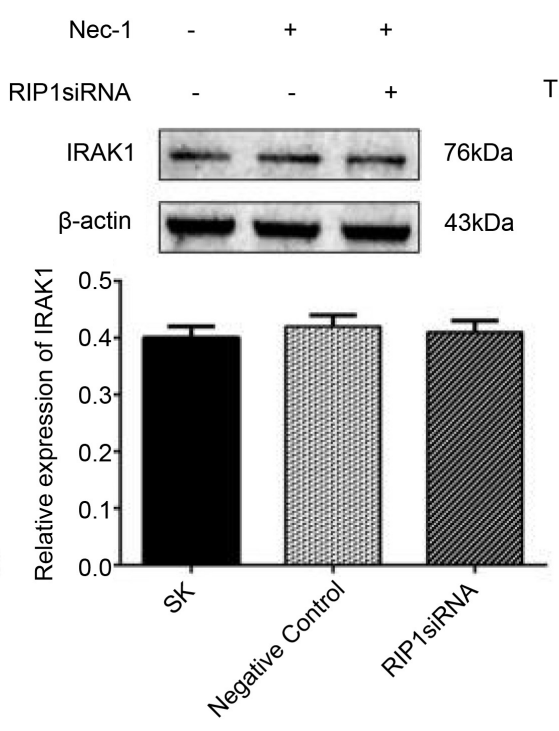

(b)

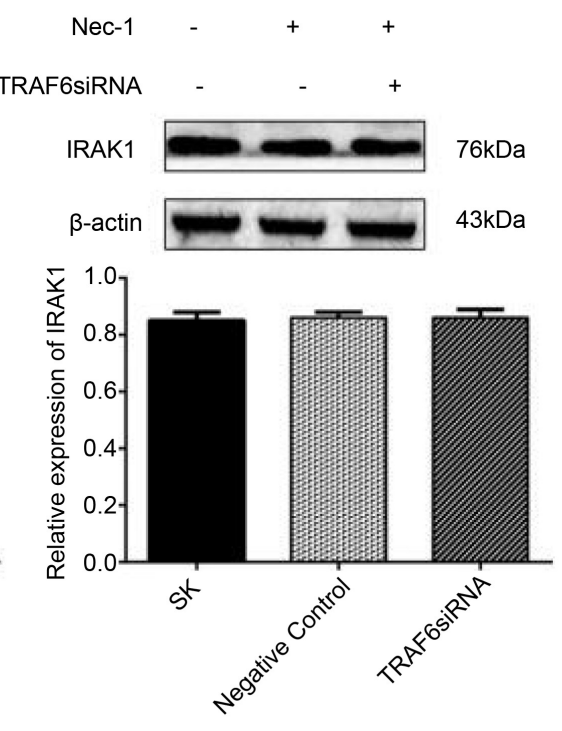

(c)

Figure 4. The expression of IRAK1 protein was detected by Western blot. (a) There was no significant change in the expression of IRAK1 protein after FADD silencing under Nec-1 intervention; (b) There was no significant change in the expression of IRAK1 protein after RIP silencing under Nec-1 intervention; (c) There was no significant change in the expression of IRAK1 protein after TRAF6 silencing under Nec-1 intervention. $(\mathrm{P}>0.05)$.

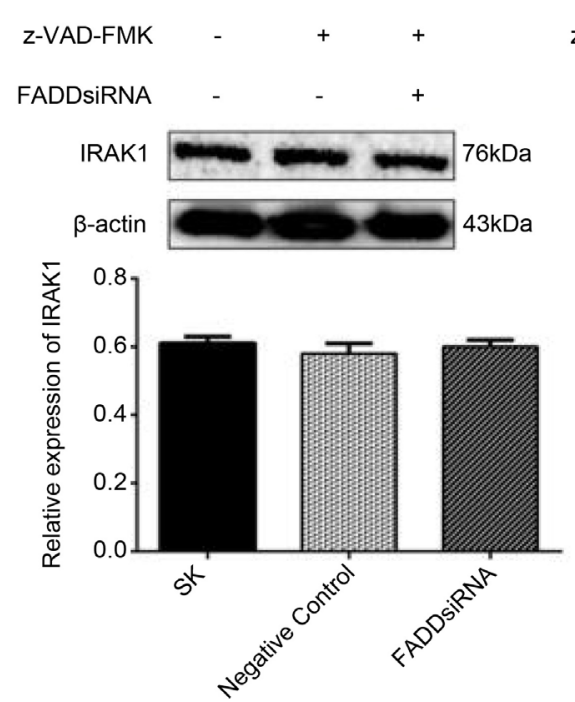

(a)

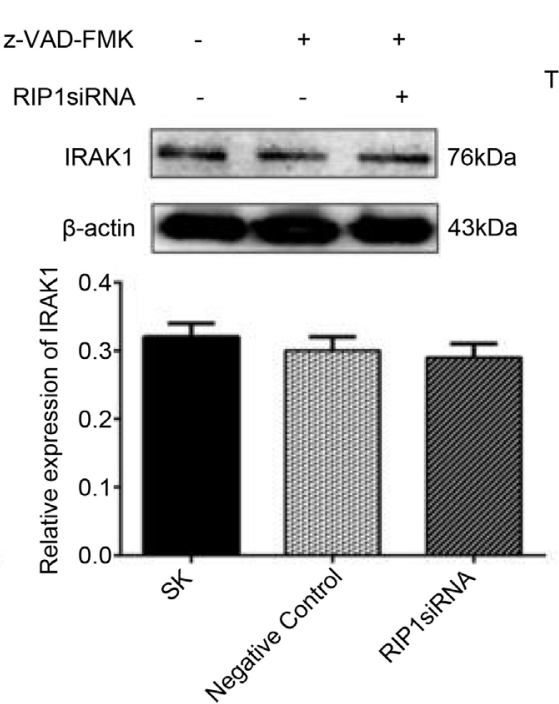

(b)

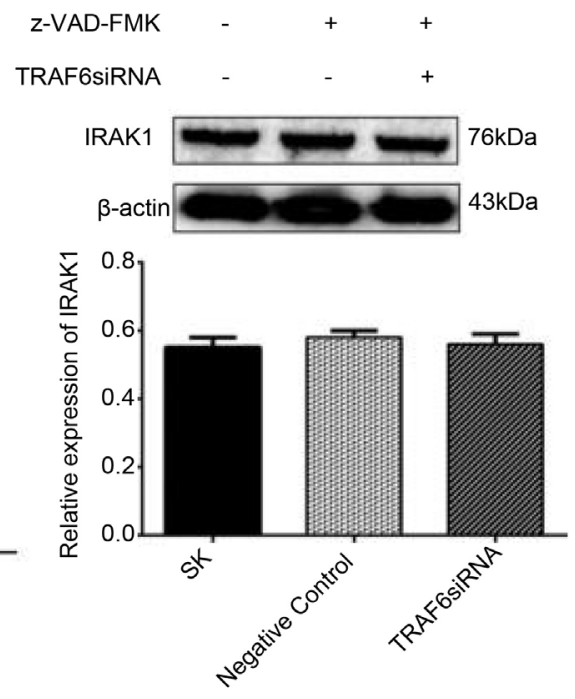

(c)

Figure 5. The expression of IRAK1 protein was detected by Western blot. (a) There was no significant change in the expression of IRAK1 protein after FADD silenced under z-VAD-FMK intervention; (b) There was no significant change in the expression of IRAK1 protein after RIP silenced under z-VAD-FMK intervention; (c) There was no significant change in the expression of IRAK1 protein after TRAF6 silenced under z-VAD-FMK intervention. $(\mathrm{P}>0.05)$.

significantly $(\mathrm{p}>0.05)$. See Figure 6. The results showed that TRAF6 gene affected the role of IRAK1 in apoptosis and necroptosis of hepatoma SK-Hep1 cells, RIP1 gene affected the role of IRAK1 in apoptosis of hepatoma SK-Hep1 cells, and FADD gene did not affect the role of IRAK1 in apoptosis and necroptosis of hepatoma SK-Hep1 cells. 


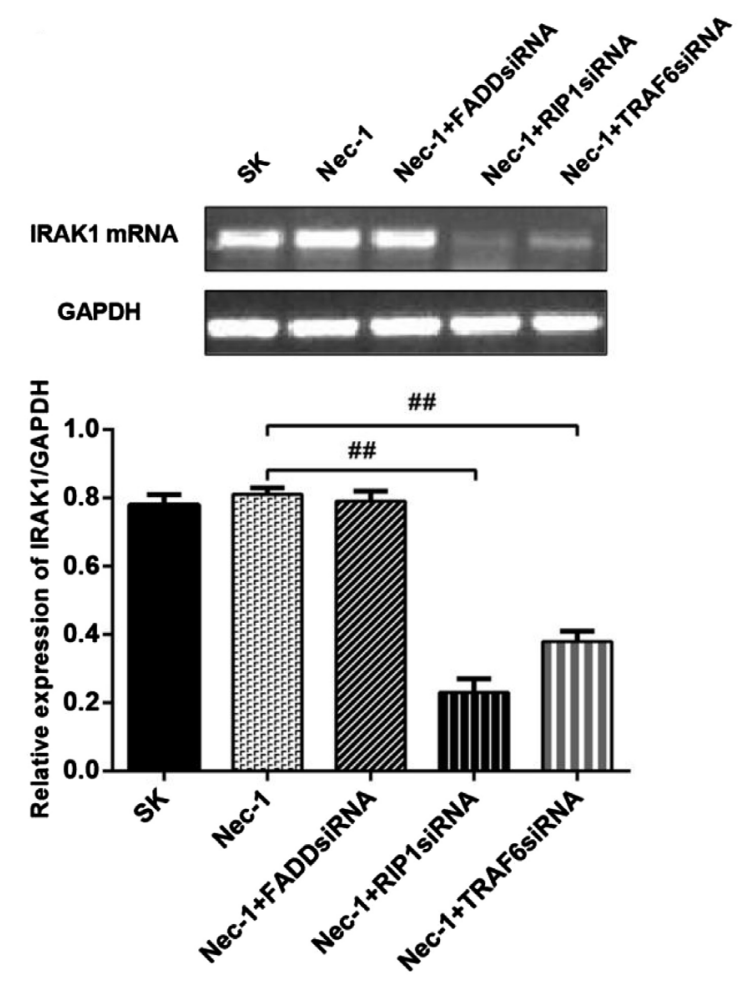

(a)

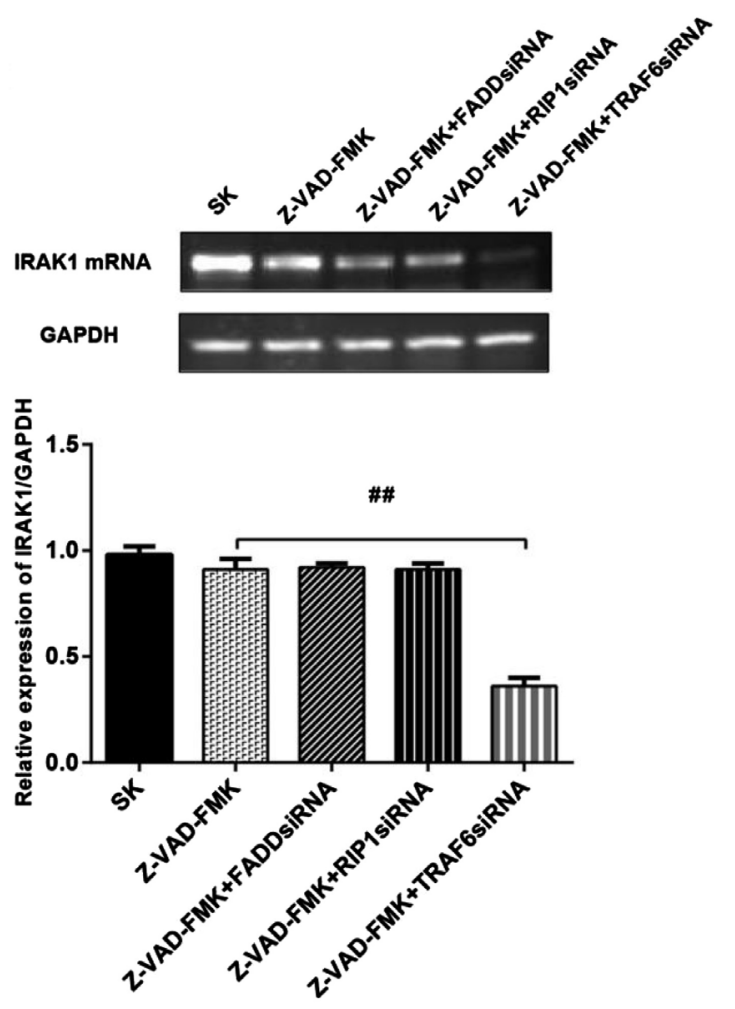

(b)

Figure 6. The expression of IRAK1 mRNA was detected by RT-PCR. (a) After RIP1 and TRAF6 were silenced under Nec-1intervention, the expression of IRAK1 mRNA was significantly decreased compared with the control group, while the expression of IRAK1 mRNA was not changed under FADD silenced; (b) After TRAF6 were silenced under z-VAD-FMK intervention, the expression of IRAK1 mRNA was significantly decreased compared with the control group, while the expression of IRAK1 mRNA was not changed under FADD and RIP1 silenced respectively. (\#\#: $\mathrm{P}<0.01$ ).

\section{Discussion}

Different signal pathways are involved in the pathogenesis of HCC, and the inflammatory signal pathway is closely related to the occurrence and development of HCC [12] [13]. The role of IRAK1 in different tumors has been widely concerned [14] [15] [16], IRAK1 is related to the formation and development of tumors, is an important tumor target [17] [18]. Infinite proliferation is an important feature of most malignant tumors, including hepatocellular carcinoma [19] [20]. Therefore, it is of great significance to study its related mechanism and seek new therapeutic strategies. The aim of this study was to investigate the effect of IRAK1 on apoptosis and necrosis of hepatoma SK-Hep1 cells. The results showed that IRAK1 could promote the apoptosis and necrosis of SK-Hep1 cells. IRAK1 may promote the progression of HCC by controlling the apoptosis and necrosis of HCC cells. Our work further identified IRAK1 inhibitors as a new therapeutic strategy for liver cancer.

Pretreatment with $\mathrm{z}$-VAD-FMK before the death ligand exposure inhibited apoptosis signals, and many types of cells could induce necroptosis and produce morphological features similar to necrosis [21] [22]. NEC-1 was the first report 
that inhibits ischemic brain damage in mice through a different mechanism from apoptosis [23]. NEC-1 was later found to be a specific inhibitor of RIP 1 [24], so cell death saved by NEC-1 could be thought of as necroptosis, and RIP 1 as a key factor in necroptosis. FADD plays an indispensable role in inducing exogenous apoptotic cell death. Recent strong evidence from in vivo studies in mice suggests that FADD plays a negative role in RIP 1 and RIP 3 dependent necroptosis [25] [26]. FADD defects are thought to inhibit caspase- 8 and subsequent apoptosis, but activate necroptosis. Under various stimuli, FADD forms a signal complex with RIP 1 and RIP 3, which plays an important role in the induction of necroptosis [27].

Apoptotic cells, at least in vivo, usually do not release their cellular contents. However, apoptotic cells do not always die silently, as they can trigger the secretion of chemokines and other immunoreactive proteins, thereby affecting the immune response to dead cells [28]. NEC-1 can not only block necroptosis, but can also modulate RIP-mediated apoptosis [29]. Therefore, Nec-1 is not a special tool to distinguish necroptosis from apoptosis. In the current study, Nec-1 and z-VAD-FMK were interfered with silenced FADD, RIP1 and TRAF6, respectively, the expression of IRAK1 protein had no significant change. However, when Nec-1 was interfered, the expression of IRAK1 mRNA decreased significantly after the silencing of RIP1 and TRAF6 genes, while the IRAK1 mRNA did not change significantly after the silencing of FADD genes; when z-VAD-FMK was interfered, the expression of IRAK1 mRNA decreased significantly after the silencing of TRAF6 genes, while the IRAK1 mRNA did not change significantly after the silencing of FADD and RIP1genes.

In addition, the results of Western blot and RT-PCR in this experiment are not completely consistent, the reason may be related to the IRAK1 transcription efficiency and protein degradation. The sensitivity of RT-PCR was higher than that of Western blot. The transcription level of mRNA was not consistent with that of protein translation. In fact, transcription and translation process itself is affected by many factors, such as phosphorylation, ubiquitin, and so on. The final synthesis of the protein is not only determined by the RNA, such as the half-life of the protein and synthesis speed can also affect the level of protein detection. Therefore, the research remains to be continued in depth.

\section{Conclusion}

In conclusions, IRAK1 gene inhibits apoptosis and necroptosis in SK-Hep1 cells. TRAF6 gene affected the role of IRAK1 in apoptosis and necroptosis, RIP1 gene affected the role of IRAK1 in apoptosis, and FADD gene did not affect the role of IRAK1 in apoptosis and necroptosis.

\section{Acknowledgements}

This study was financially supported by grants from the National Natural Foundation of China (grant no. 81160529). 


\section{Conflicts of Interest}

The authors declare no conflicts of interest regarding the publication of this paper.

\section{References}

[1] Chen, C.J., Yu, M.W. and Liaw, Y.F. (1997) Epidemiological Characteristics and Risk Factors of Hepatocellular Carcinoma. Journal of Gastroenterology and Hepatology, 12, S294-S308. https://doi.org/10.1111/j.1440-1746.1997.tb00513.x

[2] Oda, T., Tsuda, H., Scarpa, A., Sakamoto, M. and Hirohashi, S. (1992) p53 Gene Mutation Spectrum in Hepatocellular Carcinoma. Cancer Research, 52, 6358-6364.

[3] Farshid, M., Hsia, C.C. and Tabor, E. (1994) Alterations of the RB Tumour Suppressor Gene in Hepatocellular Carcinoma and Hepatoblastoma Cell Lines in Association with Abnormal p53 Expression. Journal of Viral Hepatitis, 1, 45-53. https://doi.org/10.1111/j.1365-2893.1994.tb00061.x

[4] Wan, X.W., Jiang, M., Cao, H.F., et al. (2003) The Alteration of PTEN Tumor Suppressor Expression and Its Association with the Histopathological Features of $\mathrm{Hu}-$ man Primary Hepatocellular Carcinoma. Journal of Cancer Research and Clinical Oncology, 129, 100-106.

[5] Tiniakos, D., Spandidos, D.A., Kakkanas, A., Pintzas, A., Pollice, L. and Tiniakos, G. (1989) Expression of Ras and Myc Oncogenes in Human Hepatocellular Carcinoma and Non-Neoplastic Liver Tissues. Anticancer Research, 9, 715-721.

[6] El-Serag, H.B. and Rudolph, K.L. (2007) Hepatocellular Carcinoma: Epidemiology and Molecular Carcinogenesis. Gastroenterology, 132, 2557-2576.

https://doi.org/10.1053/j.gastro.2007.04.061

[7] Akira, S. and Takeda, K. (2004) Toll-Like Receptor Signaling. Nature Reviews Immunology, 4, 499-511. https://doi.org/10.1038/nri1391

[8] Baker, B., Maitra, U., Geng, S. and Li, L. (2014) Molecular and Cellular Mechanisms Responsible for Cellular Stress and Low-Grade Inflammation Induced by a Super-Low Dose of Endotoxin. Journal of Biological Chemistry, 289, 16262-16269. https://doi.org/10.1074/jbc.M114.569210

[9] Rickard, J.A., O'Donnell, J.A., Evans, J.M., et al. (2014) RIPK1 Regulates RIPK3-MLKL-Driven Systemic Inflammation and Emergency Hematopoiesis. Cell, 157, 1175-1188. https://doi.org/10.1016/j.cell.2014.04.019

[10] Dillon, C.P., Weinlich, R., Rodriguez, D.A., et al. (2014) RIPK1 Blocks Early Postnatal Lethality Mediated by Caspase-8 and RIPK3. Cell, 157, 1189-1202.

https://doi.org/10.1016/j.cell.2014.04.018

[11] Kim, J.M., Cho, H.H., Lee, S.Y., et al. (2012) Role of IRAK1 on TNF-Induced Proliferation and NF-kB Activation in Human Bone Marrow Mesenchymal Stem Cells. Cellular Physiology and Biochemistry, 30, 49-60. https://doi.org/10.1159/000339045

[12] Li, X., Liu, C., Ip, B.C., et al. (2015) Tumor Progression Locus 2 Ablation Suppressed Hepatocellular Carcinoma Development by Inhibiting Hepatic Inflammation and Steatosis in Mice. Journal of Experimental and Clinical Cancer Research, 34, 138. https://doi.org/10.1186/s13046-015-0254-2

[13] Li, L., Xu, L., Yan, J., et al. (2015) CXCR2-CXCL1 Axis Is Correlated with Neutrophil Infiltration and Predicts a Poor Prognosis in Hepatocellular Carcinoma. Journal of Experimental and Clinical Cancer Research, 34, 129.

https://doi.org/10.1186/s13046-015-0247-1

[14] Rhyasen, G.W., Bolanos, L., Fang, J., et al. (2013) Targeting IRAK1 as a Therapeutic Approach for Myelodysplastic Syndrome. Cancer Cell, 24, 90-104. 
https://doi.org/10.1016/j.ccr.2013.05.006

[15] Zhang, X., Dang, Y., Li, P., Rong, M. and Chen, G. (2014) Expression of IRAK1 in Lung Cancer Tissues and Its Clinicopathological Significance: A Microarray Study. International Journal of Clinical and Experimental Pathology, 7, 8096-8104.

[16] Wee, Z.N., Yatim, S.M., Kohlbauer, V.K., et al. (2015) IRAK1 Is a Therapeutic Target That Drives Breast Cancer Metastasis and Resistance to Paclitaxel. Nature Communications, 6, Article No. 8746.

[17] Huang, Y.S., Misior, A. and Li, L.W. (2005) Novel Role and Regulation of the Interleukin-1 Receptor Associated Kinase (IRAK) Family Proteins. Cellular \& Molecular Immunology, 2, 36-39.

[18] Rhyasen, G.W. and Starczynowski, D.T. (2015) IRAK Signalling in Cancer. British Journal of Cancer, 112, 232-237. https://doi.org/10.1038/bjc.2014.513

[19] Feitelson, M.A., Arzumanyan, A., Kulathinal, R.J., et al. (2015) Sustained Proliferation in Cancer: Mechanisms and Novel Therapeutic Targets. Seminars in Cancer Biology, 35, S25-S54.

[20] Deng, L., Yang, H., Tang, J., et al. (2015) Inhibition of MTA1 by ERalpha Contributes to Protection Hepatocellular Carcinoma from Tumor Proliferation and Metastasis. Journal of Experimental \& Clinical Cancer Research, 34, 128.

https://doi.org/10.1186/s13046-015-0248-0

[21] Vandenabeele, P., Galluzzi, L., Vanden Berghe, T. and Kroemer, G. (2010) Molecular Mechanisms of Necroptosis: An Ordered Cellular Explosion. Nature Reviews Molecular Cell Biology, 11, 700-714. https://doi.org/10.1038/nrm2970

[22] Holler, N., Zaru, R., Micheau, O., et al. (2000) Fas Triggers an Alternative, Caspase-8 Independent Cell Death Pathway Using the Kinase RIP as Effector Molecule. Nature Immunology, 1, 489-495. https://doi.org/10.1038/82732

[23] Degterev, A., Huang, Z., Boyce, M., et al. (2005) Chemical Inhibitor of Nonapoptotic Cell Death with Therapeutic Potential for Ischemic Brain Injury. Nature Chemical Biology, 1, 112-119. https://doi.org/10.1038/nchembio711

[24] Degterev, A., Hitomi, J., Germscheid, M., et al. (2008) Identification of RIP1 Kinase as a Specific Cellular Target of Necrostatins. Nature Chemical Biology, 4, 313-321. https://doi.org/10.1038/nchembio.83

[25] Bonnet, M.C., Preukschat, D., Welz, P.S., et al. (2011) The Adaptor Protein FADD Protects Epidermal Keratinocytes from Necroptosis in Vivo and Prevents Skin Inflammation. Immunity, 35, 572-582. https://doi.org/10.1016/j.immuni.2011.08.014

[26] Lu, J.V., Weist, B.M., van Raam, B.J., et al. (2011) Complementary Roles of FAS-Associated Death Domain (FADD) and Receptor Interacting Protein Kinase-3 (RIPK3) in T-Cell Homeostasis and Antiviral Immunity. Proceedings of the National Academy of Sciences, 108, 15312-15317. https://doi.org/10.1073/pnas.1102779108

[27] Lee, E.W., Seo, J.H., Jeong, M.H., Lee, S.S. and Song, J.W. (2012) The Roles of FADD in Extrinsic Apoptosis and Necroptosis. BMB Reports, 45, 496-508. https://doi.org/10.5483/BMBRep.2012.45.9.186

[28] Cullen, S.P., Henry, C.M., Kearney, C.J., et al. (2013) Fas/CD95-Induced Chemokines Can Serve as "Find-Me" Signals for Apoptotic Cells. Molecular Cell, 49, 1034-1038. https://doi.org/10.1016/j.molcel.2013.01.025

[29] Tenev, T., Bianchi, K., Darding, M., et al. (2011) The Ripop-Tosome, a Signaling Platform That Assembles in Response to Genotoxic Stress and Loss of IAPs. Molecular Cell, 43, 432-448. https://doi.org/10.1016/j.molcel.2011.06.006 